\title{
Diarrea asociada a Clostridium difficile en niños
}

\author{
Pilar Rodríguez y José Cofré
}

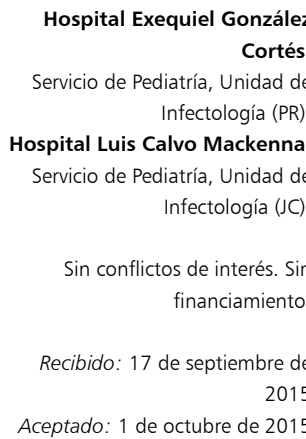

Correspondencia a: Pilar Rodríguez. piliilip@gmail.com

\section{Clostridium difficile associated diarrhea in children}

Introduction: Clostridium difficile is the most commonly isolated organism in antimicrobial and health careassociated diarrhea and is growing in relevance in community-acquired infections. It is a Gram-positive bacillus acquired via the fecal-oral route in the community and in hospital setting. Epidemiology: 0.6 to $2.1 \%$ worldwide incidence, mortality $\sim 1-5 \%$. Colonization: High rates of asymptomatic colonization in healthy people, $37 \%$ in children: its presence in stools is of controversial significance. Risk factors in children are prior exposure to antibiotics, recent hospitalization, immunosuppression or inflammatory bowel disease. Clinical manifestations: secondary to intestinal involvement due to toxin production, ranging from asymptomatic colonization to fulminant disease. Diagnosis: Clinical diagnostic criteria plus high sensitivity and specificity laboratory certification. Recommendations AAP (American Academy of Pediatrics): under 1 year, avoid routine study, only in Hirschsprung disease and/or nosocomial outbreak, 1-3 year, a (+) result suggests $C$. difficile associated diarrhea (CDAD) is possible, and in children older than 3 years interpretation is equal to adults. Management: antimicrobial suspension, oral metronidazole as first line in mild to moderate CDAD, and oral or enema vancomycin or associated with intravenous metronidazole only in severe cases. Duration 10 days. Prevention: Antimicrobial control programs and environmental management. Conclusion: Given the increasing complexity of pediatric patients it is important to deepen the knowledge on this microorganism and its clinical manifestations, as its incidence, morbidity and mortality are increasing.

Key words: Clostridium difficile, diarrhea, nosocomial, pediatrics.

Palabras clave: Clostridium difficile, diarrea, atención de salud, pediatría.

\section{Introducción}

$\longrightarrow$ lostridium difficile es un bacilo grampositivo, anaerobio obligado, que frente al estrés ambiental tiene la capacidad de formar esporas, las que liberadas al ambiente son adquiridas a través de la vía fecal oral en la comunidad y en el ambiente hospitalario ${ }^{1-3}$.

$\mathrm{Su}$ patogenicidad está dada por la producción de las toxinas $\mathrm{A}$ y $\mathrm{B}^{1,2}$. Es poco frecuente que produzca enfermedad en personas sanas con microbiota intestinal normal, pero cualquiera alteración a este equilibrio genera susceptibilidad a la infección y enfermedad, en particular en personas con factores de riesgo, a quienes puede llegar a causar la muerte ${ }^{2,3}$.

Es el microorganismo más comúnmente aislado como causa de diarrea asociada al uso de antimicrobianos y es un patógeno frecuentemente asociado a la atención de salud en la población adulta $(20-30 \%$ de los casos de diarreas nosocomiales $)^{4,5}$. Últimamente, ha sido reportado en población considerada de bajo riesgo como adultos jóvenes, niños y mujeres embarazadas ${ }^{1,6}$.

En los niños, considerados como una población de bajo riesgo para diarrea asociada a $C$. difficile (DACD), la diarrea asociada a la atención de salud es una patología completamente distinta a la del adulto. Históricamente son los virus circulantes en la comunidad, como rotavirus, norovirus y adenovirus entéricos, los responsables de este cuadro clínico. En un estudio realizado por Langley y cols., quienes estudiaron episodios de diarrea asociada a la atención de salud en niños, los virus fueron reportados con una frecuencia de $69 \%$ como agente causal ${ }^{5}$. En este mismo estudio, $C$. difficile fue aislado en $32 \%$ de los casos como agente único, no quedando claro aquí su rol patogénico ${ }^{5}$.

Es importante considerar que un alto número de personas se colonizan con $C$. difficile sin presentar manifestaciones clínicas. En adultos, en la comunidad, la colonización alcanza de 1,6 a 4\% y en la población de pacientes hospitalizados, hasta $26 \%{ }^{2}$. En niños, en especial bajo un año de edad, el significado de la presencia de $C$. difficile en las deposiciones es controversial ya que la colonización es muy superior a los adultos, alcanzando en promedio hasta $37 \%$ (rangos descritos $25-80 \%$ ), con muy escasa presentación de la enfermedad ${ }^{1-3,6}$. Incluso la presencia de toxina de $C$. difficile en este grupo etario no implica necesariamente el desarrollo de enfermedad ${ }^{2}$.

Si bien la amenaza intrahospitalaria de $C$. difficile está claramente reconocida en el adulto, su incidencia y su carga de enfermedad en niños es reportada con poca frecuencia ${ }^{5}$. Sin embargo, en los últimos años, estudios basados en grandes bases de datos en niños han demostrado que la DACD es una enfermedad presente en pediatría y que ha ido en aumento, dado la mayor complejidad de los pacientes ${ }^{6}$. Por ejemplo, Zilberberg y cols., comuni- 
caron que la tasa anual de hospitalización pediátrica por DACD en E.U.A. aumentó de 7,24 a 12,8 por 10.000 hospitalizaciones desde 1997 a 2006, datos provenientes de más de 3.700 hospitales en E.U.A. ${ }^{8}$. También, Kim y cols., en un análisis retrospectivo de 4.895 niños con infección por $C$. difficile en 22 hospitales pediátricos, demostraron un aumento de $53 \%$ en la incidencia anual desde 26 a 40 por 10.000 admisiones de 2001 a $2006^{13}$. Otro factor importante epidemiológico a considerar, es que han aumentado los casos en pacientes sin factores de riesgo, como aquellos adquiridos en la comunidad ${ }^{7}$.

Como agravante, al igual que en la población adulta, en niños se ha comunicado un aumento de casos adquiridos en la comunidad. Un estudio prospectivo realizado en Rochester, E.U.A., evaluó las muestras de niños con diarrea que consultaban al Servicio de Urgencia, encontrando que $47 \%$ de las muestras de deposiciones resultaban positivas para agentes bacterianos, de éstos $12 \%$ eran positivos para toxina de $C$. difficile ${ }^{6}$. Sí cabe comentar que no se correlacionó el hallazgo con el cuadro clínico ${ }^{6}$.

Dada la creciente complejidad de los pacientes pediátricos, es importante profundizar sobre este microorganismo y el desarrollo de enfermedad, ya que es una enfermedad cuya incidencia y morbi-mortalidad va en aumento ${ }^{1,2}$.

\section{Epidemiología}

La incidencia de DACD en el mundo, incluido Chile, varía entre 0,6 y $2,1 \%$ del total de pacientes hospitalizados, sin distinción de edad; y la tasa de mortalidad es de aproximadamente de 1 a $5 \%{ }^{12}$.

En pediatría, varios estudios han mostrado un aumento de hospitalizaciones por, e incidencia de DACD, así como también un aumento de casos en pacientes sin factores de riesgo ${ }^{3,8,9}$.

En Chile, los brotes de infecciones gastrointestinales por $C$. difficile aparecen en las notificaciones nacionales desde el año 2006 en número reducido, con un incremento brusco y significativo desde el año 2010, de manera similar a lo observado en Europa y Norteamérica ${ }^{8}$. El grupo etario más afectado fue el de 65 o más años, con $62,1 \%$ de las muestras. El número de muestras en pacientes pediátricos fue muy inferior, con menos de $1 \%{ }^{10}$.

\section{Microbiología}

Clostridium difficile es un bacilo grampositivo cuyo crecimiento óptimo es en agar sangre a $37^{\circ} \mathrm{C}$. Frente al estrés del medio, la bacteria produce endosporas que le permite tolerar condiciones ambientales extremas, resistencia a los cambios físicos y químicos como altas temperaturas, luz ultravioleta y exposición a desinfectantes que no contienen cloruros ${ }^{12,13}$. Se ha aislado por meses en centros de salud y por más de 40 días en pacientes después del egreso hospitalario ${ }^{2,9}$.

Fue identificado en 1935 como parte de la microbiota propia de recién nacidos. En 1977, fue llamado C. difficile por Hall y O' Toole para reflejar la dificultad en su aislamiento, atribuible a su crecimiento lento, en comparación con otras especies del género Clostridium ${ }^{10}$.

La transmisión es por vía fecal oral, con la ingesta de esporas que contaminan el ambiente hospitalario ${ }^{3,15}$.

\section{Patogenia}

Sus esporas son consideradas la fuente de transmisión, de infección y de persistencia en el colon del hospedero ${ }^{10}$. Entre los factores de virulencia, los dos más importantes

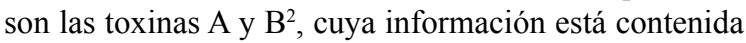
en una isla de patogenicidad donde se codifican los genes $t c d \mathrm{~A}$ y $t c d \mathrm{~B}$ de ambas toxinas, asociados a dos genes regulatorios $(t c d \mathrm{C}$ y $t c d \mathrm{R})$ y al gen productor de porina que facilita la liberación de las toxinas $(t c d \mathrm{E})^{2,17}$. Las toxinas son endocitadas y activadas en los endosomas, luego producen la glucosilación de las proteínas Rho, alterando los microfilamentos de actina y las uniones estrechas, generando un efecto citotóxico y alteración de la barrera intestinal ${ }^{16-18}$.

La toxina A particularmente genera una importante respuesta inflamatoria a través de la liberación de leucotrienos, $\mathrm{PGE}_{2} \mathrm{y}$ factor de necrosis tumoral alfa y además migración de neutrófilos ${ }^{11}$. La toxina $\mathrm{B}$, a su vez, cruza a través de la alteración de las uniones estrechas hacia el lado baso-lateral de la célula epitelial ${ }^{17}$.

La acción de ambas toxinas se traduce en un daño epitelial intenso con edema celular y secreción luminal abundante (diarrea secretoria) ${ }^{12,13}$. El daño tóxico a la mucosa colónica produce acumulación de fibrina, mucina y detritus celulares generando las pseudomembranas. Si el daño intestinal es extenso se puede producir traslocación bacteriana con bacteriemia, shock séptico e incluso la muerte.

Descrito como otro factor de virulencia, está la toxina binaria o $C$. difficile transferasa. Su unión al receptor de lipoproteína genera una traslocación hacia el citoplasma donde ribosila la actina en forma irreversible, reordenando el citoesqueleto ${ }^{14}$.

Es importante destacar la aparición de cepas epidémicas pertenecientes al ribotipo $027^{16}$, cuya presentación es en general más grave, con aumento de las tasas de infección versus colonización, mayor número de recurrencias, sepsis, megacolon tóxico, perforación intestinal y también mortalidad $^{15,16}$. Esta cepa ha entrado en la población pediátrica con tasas bajas de 10 a $19 \%$ de los aislados 
de $\mathrm{CD}^{15}$. La detección de esta cepa no es posible en la mayoría de los laboratorios y su hallazgo no influye en el manejo médico de cada paciente ${ }^{10}$.

\section{Colonización}

Un alto número de personas se colonizan con $C$. difficile sin presentar expresión clínica. En lactantes, el significado de la presencia de $C$. difficile en las deposiciones es controversial ${ }^{6,19}$. En el momento de nacer, el intestino humano es estéril; durante los primeros cinco días de vida comienza a adquirir las primeras bacterias y ya a los 12 meses su microbiota intestinal es similar al del adulto ${ }^{16}$. Los neonatos son altamente susceptibles a la colonización, tanto por transmisión ambiental como materna $^{7}$, por inmadurez intestinal y por incapacidad de suprimir el crecimiento de C. difficile ${ }^{1,2,23}$; sin embargo, no son susceptibles a la enfermedad. La causa de por qué los recién nacidos son refractarios a enfermar no está del todo claro. La hipótesis es que las toxinas productoras de enfermedad (A/B) se unen a las células del intestino grueso donde generan el daño; para esta unión es fundamental la presencia de receptores luminales para la endocitosis. Inicialmente se planteaba que los lactantes eran colonizados por cepas no toxigénicas; con posterioridad se demostró la presencia de toxinas. En un estudio en el año 2008 donde se evaluaron 42 lactantes sanos colonizados, se encontró $71 \%$ de cepas productoras de toxinas. Por lo anterior, nuevas hipótesis han planteado la carencia de los receptores necesarios para la internalización de la toxina o de la maquinaria celular necesaria, haciendo el intestino del neonato y lactante resistentes a la capacidad de daño de la bacteria ${ }^{2}$.

En niños de uno a seis meses la colonización disminuye hasta $30 \%{ }^{1-3}$, llegando a alrededor de $10 \%$ en lactantes sanos de 1 año ${ }^{1-3}$. A los 3 años alcanza la frecuencia del adulto no hospitalizado, eso es $3 \% \%^{2,3}$. La desaparición de C. difficile de la microbiota intestinal después del año de vida tendría mayor relación con cambios nutricionales y fisiológicos a favor de los microorganismos que compiten con el crecimiento de C. difficile ${ }^{23}$.

Otros factores generales evaluados son:

Vía del parto: Estudios que evalúan la presencia de $C$. difficile en deposiciones en niños nacidos no han mostrado diferencias en la colonización según cuál fuera la vía de resolución del mismo: vaginal, cesárea o si hubiera habido una instrumentalización durante el parto (fórceps) ${ }^{22}$. Lamont y cols., encontraron tasas de colonización de 30\% en parto vaginal versus $37,5 \%$ en cesáreas, sin mayor significancia estadística ${ }^{22}$.

Estadia hospitalaria: Este factor ha demostrado aumentar la tasa de colonización en recién nacidos, con un aumento proporcional según la cantidad de días de hospitalización ${ }^{17}$. La forma de adquisición del microorganismo más probable sería desde las unidades de neonatología, más que de la madre ${ }^{6,24}$. Para este propósito Meisel-Mikolajczyk y cols., tomaron hisopados vaginales y rectales a 183 madres, muestras fecales a sus recién nacidos y 94 muestras ambientales. No encontraron muestras positivas de las madres, tampoco las hallaron al día uno de vida del neonato, pero sí encontraron $17 \%$ de neonatos colonizados al día cuatro. De las muestras ambientales, $13 \%$ fueron positivas para $C$. difficile ${ }^{18}$. Al genotipificar las muestras, encontraron dos genotipos; el genotipo 1 fue encontrado en 13/31 recién nacidos positivos y en 10/11 de las muestras ambientales, lo que implica que éstas fueron adquiridas del ambiente de la unidad de neonatología. Dado que las muestras maternas resultaron negativas, es menos probable que la adquisición fuese por esa vía ${ }^{24}$.

Lactancia materna versus artificial: Los niños alimentados con leche materna tienen tasas menores de colonización por $C$. difficile, 6-20\%, comparados con aquellos con alimentación artificial quienes alcanzan tasas de $49-66 \%{ }^{23}$. El mecanismo por el cual la lactancia natural previene la colonización no está del todo claro. Se plantean distintas hipótesis, por ejemplo, que existe un factor inhibidor del crecimiento de $C$. difficile en la leche materna ${ }^{19}$; también, que los niños alimentados con leche materna tienen un $\mathrm{pH}$ fecal menor a los con fórmula, lo que inhibiría el crecimiento, facilitaría la esporulación y reduciría la producción de toxinas por las formas vegetativas del microorganismo ${ }^{20}$. Otro planteamiento es que las proteínas contenidas en la leche materna inhiben la unión de la toxina A a receptores colónicos; en hamsters, este efecto inhibitorio alcanzó una intensidad de $90 \%{ }^{21}$. Por último, la leche materna estimularía el desarrollo de microorganismos que inhiben la colonización por $C$. difficile, como Bifidobacterium spp. y Lactobacillus spp..$^{22}$.

En resumen, la resistencia de los niños a la enfermedad estaría relacionada con la ausencia de la maquinaria para unir y activar la toxina, con los componentes protectores de la leche materna y con la competencia con la microbiota comensal $^{20-24}$.

Cabe destacar que, a pesar de la alta colonización intestinal por $C$. difficile, la tasa de DACD antes del año de vida es muy infrecuente. Sin embargo, hay reportes donde sí se pone en evidencia la gravedad que puede alcanzar la enfermedad. Benson y cols., realizaron un estudio retrospectivo donde incluyeron pacientes pediátricos hospitalizados entre julio de 2001 y mayo de 2006. De un total de 513 pacientes, con 827 muestras positivas para $C$. difficile, 313 (61\%) eran mayores de dos años, 69 (13,5\%) tenían bajo tres meses de edad, y de éstos, 25 (4,8\%) tenían menos de seis semanas de edad. Respecto a la expresión clínica, encontraron 31 muestras positivas en 22 pacientes de la unidad de cuidados intensivos neonatales. De éstos, 
$20(91 \%)$ tenían patología gastrointestinal de base, cinco (23\%) fueron sometidos a resección de intestino delgado y cinco (23\%) fueron diagnosticados con colitis. Cuatro (18\%) requirieron colostomía o colectomía y 11 (50\%) tuvieron sospecha o certeza de enterocolitis necrosante ${ }^{26}$.

Por todo lo anterior, hay que mantener la atención en la evolución epidemiológica de esta enfermedad, dado la mayor complejidad de los pacientes que tienen un riesgo potencial mayor, sumado al aumento de la incidencia, no sólo en neonatos, sino también en otros grupos etarios.

\section{Factores de riesgo para DACD en niños}

La presencia de co-morbilidades es frecuente en niños con DACD, ya que se ven expuestos a mayor número de hospitalizaciones, mayor uso de antimicrobianos, inmunosupresores y otros factores que alteran la microbiota intestinal ${ }^{2}$.

En una cohorte retrospectiva de pacientes hospitalizados por DACD comunicada por Kim y cols., $67 \%$ tenía al menos una co-morbilidad asociada, las más frecuentes eran cardiopatías congénitas en neonatos y cáncer en escolares entre 5-17 años ${ }^{3}$.

Los factores de riesgo para el desarrollo de DACD en niños pueden ser divididos en factores modificables y en aquellos no modificables ${ }^{22}$.

\section{Factores modificables}

- Exposición previa a antimicrobianos: Los antimicrobianos modifican la microbiota intestinal normal creando un nicho para $C$. difficile. En un estudio canadiense entre 2000 y 2003, se estudiaron 200 niños con DACD, resultando que $75 \%$ había utilizado antimicrobianos en los dos meses previos ${ }^{23}$. La mayoría de las familias de antimicrobianos ha sido asociada al desarrollo de esta enfermedad ${ }^{2}$.

- Supresión de la acidez gástrica: La supresión de la acidez permite la supervivencia de las esporas al paso gástrico. En un estudio caso control en 68 niños, Turco y cols., obtuvieron un OR 4,5 para el uso de bloqueadores de bomba de protones (IC 95\% 1,4-14,4) ${ }^{27}$.

- Uso prolongado de sonda nasogástrica, sonda nasoyeyunal o gastrostomía: La instalación de dispositivos de alimentación genera disrupciones en la mucosa y el mismo dispositivo puede introducir esporas a través de la contaminación de las manos del personal o de fórmulas alimentarias. En un estudio caso control de 95 niños con DACD, la probabilidad de tener gastrostomía o yeyunostomía era de 3,32 en aquellos que desarrollaron DACD (IC 95\% 1,71-6,42) ) $^{27,30}$.

- Hospitalización reciente: Hasta los seis meses pre$\operatorname{vios}^{8,9}$.

\section{Factores no modificables}

- Cáncer/Quimioterapia: El riesgo aumentado puede ser atribuido a la enfermedad de base, a la actividad antimicrobiana de la quimioterapia, al uso prolongado de antibacterianos de amplio espectro, al daño de la mucosa intestinal relacionado con la toxicidad de la quimioterapia y a la hospitalización prolongada. En un estudio retrospectivo de 4.051 casos de DACD pediátrico, la incidencia en niños con cáncer versus sin cáncer fue de 17,7 versus 1,1 casos por 1.000 altas, respectivamente ${ }^{27}$. Otro estudio, donde se evaluaron los factores de riesgo para DACD grave en dos hospitales pediátricos, demostró que la presencia de cáncer y/o terapia inmunosupresora estaba en $21 \%$ de todos los casos de DACD y que todos los casos de niños con cáncer presentaron formas graves de evolución ${ }^{9}$.

- Trasplante de precursores hematopoyéticos: Experiencias clínicas revelan que las tasas de DACD en pacientes que han recibido trasplante de precursores hematopoyéticos llega a ser nueve veces mayor comparados con la población general y 1,4 veces superior a aquella en pacientes oncológicos sometidos a quimioterapia (24 vs 2,6 vs 16,8 x 10.000 día paciente, respectivamente) ${ }^{24}$.

- Hipogamaglobulinemia: Por déficit congénito o adquirido de anticuerpos protectores contra antitoxinas. La evidencia sólo se limita a datos observacionales ${ }^{27}$.

- Trasplante de órganos sólidos: Al igual que en adultos, el riesgo mayor es en los primeros tres meses post trasplante (hígado, corazón, pulmón o riñón). El riesgo está dado por las hospitalizaciones prolongadas, la inmunosupresión intensa y el frecuente uso de antimicrobianos de amplio espectro. En un estudio caso-control de 95 niños con DACD, los pacientes receptores de trasplante tenían un OR 8,09 (IC 95\% 2,1-31,1).

- Enfermedad de Hirschsprung: La enterocolitis asociada a enfermedad de Hirschsprung (EAEH) es la complicación más grave y potencialmente fatal de esta enfermedad, con morbilidad y mortalidad significativas. No existe evidencia suficiente que relacione un microorganismo causal específico con la EAEH, pero es $C$. difficile el microorganismo encontrado con mayor frecuencia y en mayor concentración en las heces de pacientes con EAEH comparados con grupos controles. Por el contrario, en otros estudios son otros los microorganismos que han mostrado mayor prevalencia, como rotavirus, Escherichia coli y Cryptosporidium parvum. Lo anterior sugiere que la susceptibilidad a la infección, dada por una barrera mucosa deficiente, alteraciones en la secreción de moco y en la producción de inmunoglobulinas, podría ser más importante que la detección de un microorganismo responsable específico ${ }^{25}$. 
- Enfermedad inflamatoria intestinal: Numerosos estudios han comunicado un aumento en la incidencia de DACD en niños con enfermedad inflamatoria intestinal (EII). La tasa anual de DACD en una cohorte de niños con EII hospitalizados fue de 7,4\%; pacientes que además presentaban una mayor tasa de recurrencia y de fracaso de tratamiento ${ }^{26}$. Se observó la asociación de DACD con una mayor gravedad en la evolución de EII, mayor necesidad de hospitalización y escalada en terapia inmunosupresora post-infección ${ }^{27}$.

- Fibrosis quística: El mayor riesgo no está claro si es por la enfermedad de base o por el uso prolongado y repetido de antimicrobianos de amplio espectro ${ }^{27}$.

\section{Cuadro clínico de la DACD}

El período de incubación, desde la exposición hasta el comienzo de los síntomas de DACD no se conoce con certeza. En tres estudios ha sido estimado en promedio de dos a tres días ${ }^{33}$. Se ha evaluado que el riesgo de DACD es mayor entre los 3 y 14 días desde el inicio de la primera terapia antimicrobiana. Este riesgo persiste los cinco días posteriores a la suspensión de la terapia y es mayor mientras el tratamiento sea más largo, pero cabe destacar que incluso dosis unitarias de antimicrobianos entrañan un riesgo de desarrollo de $\mathrm{DACD}^{28}$. La adquisición de la infección es, en general, desde el ambiente hospitalario, a través de la ingesta de esporas. Se le considera una infección intrahospitalaria si los síntomas aparecen después de las $48 \mathrm{~h}$ de la admisión o antes de cuatro semanas desde el alta. Su origen es indeterminado si es entre 4 y 12 semanas desde el alta y adquirida en la comunidad si el paciente no ha sido hospitalizado o ya han pasado más de 12 semanas desde el egreso ${ }^{34}$.

La expresión clínica es el resultado del compromiso intestinal secundario a la producción de toxinas.

La definición de caso incluye diarrea (tres o más deposiciones no formadas en un período de $24 \mathrm{~h}$ ) y ya sea test positivo para toxina de $C$. difficile o hallazgos endoscópicos compatibles con pseudomembranas.

El espectro clínico va desde la colonización asintomática hasta la enfermedad fulminante.

La enfermedad se clasifica en leve, moderada, grave y grave con complicaciones ${ }^{34}$. Los cuadros leves a moderados incluyen la diarrea con o sin sangre y mucosidad, sin cumplir criterios de gravedad ${ }^{9,29}$.

Los casos graves pueden o no presentar complicaciones como íleo paralítico o megacolon tóxico, con deshidratación, alteraciones hidro-electrolíticas, perforación intestinal, hipotensión arterial, falla renal, sepsis y muerte ${ }^{30}$. Kim y cols. ${ }^{36}$, entregaron por primera vez definiciones para enfermedad grave en pediatría considerando dos escenarios clínicos:
- Criterios de enfermedad grave: aquellos pacientes con DACD y dos o más de los siguientes criterios:

- Leucocitos $>15.000 \mathrm{o}<5.000 / \mathrm{mm}^{3}$.

- Albuminemia $<2,5 \mathrm{mg} / \mathrm{dL}$.

- Creatininemia elevada para la edad.

- Disentería o hemorragias ocultas en deposiciones.

- Fiebre $>38,5^{\circ} \mathrm{C}$ por más de un día, en los siete días previos al diagnóstico.

- Presencia de complicaciones, al menos una de las siguientes condiciones: colitis pseudomembranosa en endoscopia o histopatología, intervención quirúrgica como consecuencia de DACD, perforación gastrointestinal, megacolon tóxico, neumatosis intestinal o necesidad de ingreso a unidad de cuidados intensivos al momento del diagnóstico o reingreso en los dos días posteriores al diagnóstico.

Los mismos autores definen como falla al tratamiento, la necesidad de cambio de terapia desde metronidazol a vancomicina oral durante el tratamiento inicial. Y definen como recaída a cualquier recurrencia de síntomas durante las siguientes ocho semanas de seguimiento.

Este grupo analizó 82 pacientes con DACD, 48 desarrollaron enfermedad grave. Como factores de riesgo, se encontró la edad con una mediana de 5,93 $(1,78-12,16)$ en el grupo de mayor gravedad ( 0,012$)$, el uso de tres clases de antimicrobianos en los 30 días previos al desarrollo de le enfermedad (OR 3,95 $(1,19-13,11))$ y, si bien todos los pacientes portadores de alguna patología oncológica tuvieron presentaciones graves, este factor no resultó significativo (OR 2,9 (0,94-8,91)). No hubo diferencias significativas entre el grupo de enfermedad grave y no grave, respecto al fracaso al tratamiento y a las recaídas. Hubo un caso fatal.

En relación a otros estudios sobre evolución clínica, Suh y cols., describieron un grupo de 97 niños con DACD, con dos fallecidos y un paciente que requirió colectomía ${ }^{31}$. Kim y cols. incluyeron 4.500 niños con DACD, de éstos 61 pacientes $(1,2 \%)$ requirieron colectomía, con una mortalidad global de $4 \%$.

Se han descrito complicaciones extra-intestinales poco frecuentes de la DACD como bacteriemia, peritonitis, absceso perianal, infección de sitio quirúrgico, infecciones músculo-esqueléticas incluidas artritis séptica y osteomielitis $^{32}$. En niños, estas complicaciones son muy infrecuentes. Sí se ha descrito casos asociados a prolapso rectal $^{33}$ y síndrome hemolítico urémico ${ }^{34}$.

\section{Diagnóstico}

Para la certificación diagnóstica de DACD existe una amplia variedad de ensayos que se utilizan en forma individual o en combinación como un algoritmo de de- 
tección rápida, con elevada sensibilidad y especificidad ${ }^{15}$. Sin embargo, es fundamental la combinación de criterios clínicos y de laboratorio. El estudio microbiológico debe solicitarse sólo si el paciente presenta deposiciones diarreicas. El aislamiento del microorganismo en las deposiciones no es útil como diagnóstico clínico al igual que el estudio de deposiciones en pacientes asintomáticos ${ }^{3}$.

El objetivo de un buen método de diagnóstico de laboratorio es que sea rápido, con alta sensibilidad y especificidad $^{15}$.

Los métodos para el diagnóstico de $C$. difficile son:

- Cultivo para aislamiento de cepas toxigénicas: Tiene una elevada especificidad y sensibilidad; sin embargo, es un proceso lento ya que requiere el aislamiento de colonias de $C$. difficile y evaluar su toxicidad en cultivo de tejidos, lo que demora varios días. Estos ensayos son útiles para validar nuevas técnicas ${ }^{15}$.

- Detección de productos del agente o efectos de dichos productos directo de las deposiciones:

- Ensayo de citotoxicidad: muestra el efecto citopático de la toxina B en cultivos celulares. Es la prueba de mayor especificidad (97 a 100\%) y considerada la técnica de referencia por mucho tiempo; sin embargo, sus desventajas son numerosas: no está estandarizada, requiere experiencia en la técnica del cultivo celular, infraestructura adecuada y un tiempo de procesamiento prolongado $(48 \text { a } 72 \mathrm{~h})^{40}$.

- Detección de toxinas: los inmunoensayos enzimáticos (EIA) para toxinas $\mathrm{A} y / 0 \mathrm{~B}$, son rápidos $\mathrm{y}$ relativamente baratos, pero su sensibilidad y especificidad son subóptimas $(72 \% \text { y } 97 \%)^{3}$ y presentan un bajo valor predictor positivo, sobre todo en niños cuya tasa de enfermedad es baja, por lo que la Sociedad de Epidemiología en Salud de América (SHEA) y la Sociedad de Enfermedades Infecciosas de América (IDSA) ya no recomiendan su uso ${ }^{9,33}$.

- Detección de glutamato deshidrogenasa (GDH): es un antígeno común presente en las cepas de $C$. difficile, tanto toxigénicas como no toxigénicas. Es un test muy sensible pero requiere confirmación por métodos específicos, por lo que se sugiere sea utilizado como parte de algoritmos diagnósticos, para abaratar costos. Sin embargo, tiene variabilidad en su rendimiento llegando hasta $15 \%$ de falsos negativos ${ }^{15}$.

- Pruebas de detección de sus genes, principalmente toxinas (tcdA, $\boldsymbol{t c d} \mathbf{B})$ : El uso de test de amplificación de ácidos nucleicos (en inglés nucleic acid amplification test-NAATs), como la RPC, aprobados por la FDA, han emergido y se han establecido como una alternativa rápida, sensible y específica, pero de mayor costo posicionándose como los favoritos para el diagnóstico ${ }^{3}$. En un estudio de sensibilidad comparativa entre RPC en tiempo real (RPC-TR) para toxina A/B versus EIA, la sensibilidad por RPC-TR fue superior en 95 versus $35 \%$ y la especificidad fue de $100 \%$ con ambas técnicas ${ }^{35}$. Entre los métodos más prometedores está el LAMP (i.e., loop mediated isothermal amplification), amplificación de 204 pares de bases del gen $t c d \mathrm{~A}$, con un tiempo de respuesta de $1 \mathrm{~h}$ y una sensibilidad y especificidad de $98 \%$. Otra alternativa es la RPC-TR para detectar directamente el gen $t c d \mathrm{~B}$, que codifica para la toxina $\mathrm{B}$, en muestras de deposiciones ${ }^{15}$.

En general, se recomienda el uso de algoritmos en la detección de $C$. difficile, ya que permiten utilizar como primera aproximación métodos fáciles de implementar y altamente sensibles, como GDH, pero con una menor especificidad en comparación con los métodos confirmatorios y altamente específicos de detección, como la biología molecular. Sí es importante considerar que el costo de errar en el diagnóstico de DACD por medio de métodos de tamizaje, en especial en niños, es demasiado elevado; por lo que se sugiere fuertemente el uso de métodos moleculares ${ }^{15}$.

\section{Estudio recomendado en niños}

En el año 2010 la SHEA y la IDSA publicaron guías prácticas sobre el manejo de DACD en adultos, las que no se refieren a pediatría.

La AAP (American Academy of Pediatrics) hizo una declaración donde recomendó:

- En lactantes bajo un año de edad ${ }^{3}$ :

- Evitar el estudio rutinario.

- Estudiar sólo a aquellos con enfermedad de Hirschprung $\mathrm{u}$ otras alteraciones graves de la motilidad intestinal.

- Estudiar en situación de brote nosocomial.

- Siempre estudiar primero causas virales en niños bajo los 3 años.

- En pacientes con resultados positivos buscar siempre una etiología alternativa.

- Niños entre uno y tres años ${ }^{3}$ :

- Estudiar niños con diarrea, donde otras causas hayan sido descartadas.

- Niños con factores de riesgo realizar estudio por fases.

- Pacientes con resultado positivo significa DACD posible.

- Niños mayores de tres años el estudio debe ser similar al de adultos ${ }^{3}$. 
- Hallazgos endoscópicos sugerentes de colitis pseudomembranosa (pseudo-membranas o mucosa rectal friable e hiperémica) son suficientes para el diagnóstico de enfermedad por $C$. difficile a cualquier edad ${ }^{3}$.

- Nunca repetir el estudio para evaluar respuesta a tratamiento ${ }^{3}$.

- Esperar un intervalo de cuatro semanas para el estudio de recurrencia ${ }^{3}$.

\section{Manejo}

El primer paso en el manejo de la DACD es la suspensión de los antimicrobianos en uso, lo que puede ser suficiente para controlar los síntomas.

En aquellos pacientes con enfermedad moderada a grave se debe comenzar con tratamiento empírico desde la sospecha diagnóstica.

La AAP recomienda el uso de metronidazol como primera línea en niños con DACD leve a moderada, en base a su eficacia, costo y control de antimicrobianos.

En adultos, la SHEA y la IDSA recomiendan el uso de vancomicina oral en casos graves, fracaso de metronidazol o contraindicación de este último. No existen estudios comparativos en niños ${ }^{9}$.

El uso de vancomicina oral, en enema o asociada a metronidazol intravenoso está indicado por la AAP sólo en casos de enfermedad grave, como por ejemplo, en pacientes neutropénicos o en pacientes con enfermedad inflamatoria intestinal o enfermedad de Hirschsprung.

Datos observacionales muestran que como primera línea se usa metronidazol en 53-63\% de los niños, y vancomicina oral en 3,5 a $30 \%{ }^{4}$. La duración de la terapia es de 10 días $^{3}$. No es necesario efectuar controles microbiológicos al término del tratamiento, ya que con alta frecuencia los pacientes continúan excretando la toxina de $C$. difficile y los cultivos continúan siendo positivos ${ }^{3}$.

En cuanto a la susceptibilidad antimicrobiana, en un estudio realizado por el Instituto de Salud Pública de Chile con cepas de los años 2012-2013 (n: 410) se observó que todas las cepas estudiadas fueron sensibles a metronidazol. Respecto a vancomicina, se observó una susceptibilidad reducida en una cepa confirmada el año 2012, el resto de las cepas analizadas resultaron sensibles a este antimicrobiano ${ }^{8}$.

Hasta $30 \%$ de los adultos tratados presentan recurrencia luego de la suspensión del tratamiento, ya sea por recaída de la misma cepa o por re-infección. En general, responden al mismo tratamiento. Pero ya en un tercer episodio no se recomienda el uso de metronidazol ${ }^{3,4}$. No existen mayores datos en niños.

Otros tratamientos usados en adultos en caso de recurrencias, como rifaximina, no ha sido estudiada en niños y tiene alta frecuencia de desarrollo de resistencia intra-tratamiento. Fidaxomicina no ha sido aprobada por la FDA para su uso en niños ${ }^{4}$.

El uso de probióticos no está recomendado por la AAP para la DACD, dado que no existen estudios controlados en niños ${ }^{3}$. Una revisión de la Cochrane Library 2013 sobre el tema concluye que existe moderada evidencia sobre el efecto protector de los probióticos para DACD pero no reduce la incidencia de la enfermedad. En términos absolutos se prevendrían 35 casos por cada 1.000 pacientes que lo recibieron en forma profiláctica. Los probióticos demostraron ser seguros y efectivos como complemento al tratamiento antimicrobiano sólo en pacientes inmunocompetentes $^{36}$.

Se recomienda evitar el uso de antiperistálticos, ya que aumentan el riesgo de megacolon tóxico ${ }^{3}$.

El trasplante fecal está descrito en reporte de casos en pediatría, con buenos resultados. Se evaluaron 10 niños con recurrencia de DACD que recibieron una infusión única de microbioma fecal de un donante sano, dos a través de una sonda nasogástrica o vía colonoscópica. Nueve pacientes $(90 \%)$ tuvieron resolución de sus síntomas con seguimiento entre un mes y cuatro años, sin presentar eventos adversos durante o después de la infusión ${ }^{24}$.

\section{Prevención}

La infección por $C$. difficile puede ser prevenida disminuyendo la frecuencia, duración y número de antimicrobianos utilizados, a través de programas de control de antimicrobianos ${ }^{9}$.

Dado su transmisión fecal-oral, el manejo ambiental es fundamental ${ }^{3}$. Para prevenir su diseminación son importantísimas las medidas de precauciones estándar es, el lavado de manos con agua y jabón, evitar el uso de alcohol gel y precauciones de contacto, las que duran hasta que se haya resuelto la diarrea ${ }^{3}$.

El aseo ambiental debe realizarse con soluciones cloradas, ya que las esporas no son eliminadas por otros desinfectantes, cobrando vital importancia la educación tanto del personal de la unidad como de los servicios de limpieza sobre la forma de aseo terminal en casos de DACD $^{9}$

\section{Resumen}

Introducción: Clostridium difficile, microorganismo más común en diarrea asociada a antimicrobianos, a atención de salud y en aumento en la comunidad es un bacilo grampositivo adquirido vía fecal oral en la comunidad y en el ambiente hospitalario. Epidemiología: Incidencia mundial 0,6-2,1\%, mortalidad 1-5\%. Colonización: Alta colonización asintomática en personas sanas, niños 37\%, 


\section{Infectología al Día}

su presencia en las deposiciones es controversial. Factores de riesgo en niños: exposición previa a antimicrobianos, hospitalización reciente, inmunosupresión o enfermedad inflamatoria intestinal. Clínica: Compromiso intestinal secundario a la producción de toxinas. Puede variar desde una colonización asintomática hasta enfermedad fulminante. Diagnóstico: La certificación diagnóstica requiere de un criterio clínico más laboratorio rápido, con elevada sensibilidad y especificidad. Recomendaciones de American Academy of Pediatrics son en lactantes bajo un año, evitar estudio rutinario, sólo enfermedad de Hirschprung y/o brote nosocomial, entre 1-3 años; un resultado (+) indica DACD posible y en mayores de 3 años los criterios son igual a adultos. Manejo: Suspensión de antimicrobianos, metronidazol ev como primera línea en niños con DACD leve a moderada y vancomicina oral, enema o asociada a metronidazol intravenoso sólo en casos graves. Duración 10 días. Prevención: Control de antimicrobianos y manejo ambiental. Conclusión: Dada la creciente complejidad de pacientes pediátricos, es importante profundizar sobre este microorganismo y el desarrollo de enfermedad, ya que su incidencia y morbi-mortalidad van en aumento.

\section{Referencias bibliográficas}

1.- Sammons J S, Toltzis P, Zaoutis T E. Clostridium difficile infection in children. JAMA Pediatr 2013; 167: 567-73.

2.- Loo V G, Bourgault A M, Poirier L, Lamothe F, Michaud S, Turgeon N, et al. Host and pathogen factors for Clostridium difficile infection and colonization. N Engl J Med 2011; 365: 1693703.

3.- Schutze G E, Willoughby R E. Committee on Infectious Diseases; American Academy of Pediatrics. Clostridium difficile infection in infants and children. Pediatrics 2013; 131: 196-200.

4.- Schutze G, Willoughby R. Policy offers: Recommendations on managing $C$. difficile infections in pediatric patient. AAP News 2013; 34: 1 .

5.- Langley J M, LeBlanc J C, Hanakowski M, Goloubeva O. The role of Clostridium difficile and viruses as cause of nosocomial diarrhea in children. Infect Control Hosp Epidemiol 2002; 23: 660-4.

6.- Khanna S, Baddour L, Huskins W C, Kammer P P, Faubion W A, Zinsmeister A R, et al. The epidemiology of Clostridium difficile infection in children: a population-based study. Clin Infect Dis 2013; 56: 1401-6.

7.- Kim J, Smathers S A, Prasad P, Leckerman K H, Coffin S, Zaoutis T. Epidemiological features of Clostridium difficile-associated disease among inpatients at children's hospitals in the United States, 2001-2006. Pediatrics 2008; 122: 126670.

8.- Instituto de Salud Pública. Vigilancia de Laboratorio de Clostridium difficile. Chile, 2012-2013. Boletín ISP. Abril de 2014; Vol 4 (N ${ }^{\circ}$ ) http://www.ispch.cl/sites/default/files/ Boletin Clostridium difficile 0.pdf

9.- Centers for Disease Control and Prevention. Clostridium difficile infection. Disponible en: http://www.cdc.gov/HAI/organisms/cdiff/Cdiff. (Consultado: 1 de abril de 2014).

10 .- Hernández-Rocha $\mathrm{C}$, Naour $\mathrm{S}$, Álvarez-Lobos M, Paredes-Sabj D. Infecciones causadas por Clostridium difficile: una visión actualizada. Rev Chilena Infectol 2012; 29 (4): 434-45.

11 .- Pothoulakis C, Lamont J T. Microbes and microbial toxins: paradigms for microbial mucosal interactions II. The integrated response of the intestine to Clostridium difficile toxins. Am J Physiol Gastrointest Liver Physiol 2001; 280: G178-83.

12 .- Rineh A, Kelso M J, Vatansever F, Tegos G P, Hamblin M R. Clostridium difficile infection: molecular pathogenesis and novel therapeutics. Exp Rev Anti Infect Ther 2014; 12: 131-50.

13 .- Voth D E, Ballard J D. Clostridium difficile toxins: mechanism of action and role in disease. Clin Microbiol Rev 2005; 18: 247-63.

14 .- Barra-Carrasco J, Hernández-Rocha C, Ibáñez P, Guzmán-Durán A, Álvarez-Lobos M, Paredes-Sabja D. Esporas de Clostridium difficile y su relevancia en la persistencia y transmisión de la infección. Rev Chilena Infectol 2014; 31: 694-703.

15.- Toltiz P, Kim J, Dul M, Zoltanki J, Smathers $\mathrm{S}$, Zaoutis T. Presence of the epidemic North American pulsed field type 1 Clostridium difficile strain in hospitalized children. J Pediatr 2009; 154: 607-8.

16.- Jangi S, Lamont J T. Asymptomatic colonization by Clostridium difficile in infants: implications for disease in later life. J Pediatr Gastroenterol Nutr 2010; 51: 2-7.

17.- McFarland L V, Mulligan M E, Kwok R Y, Stamm W E. Nosocomial acquisition of Clostridium difficile infection. N Engl J Med 1989; 320: 204-10.

18 .- Martirosian G, Kuipers S, Verbrugh H, van Belkum A, Meisel-Mikolajczyk F. PCR ribotyping and arbitrarily primed PCR for typing strains of Clostridium difficile from a Polish maternity hospital. J Clin Microbiol 1995; 33: 2016-21.

19.- Benno Y, Sawada K, Mitsuoka T. The intestinal microflora of infants: composition of fecal flora in breast-fed and bottle-fed infants. Microbiol Immunol 1984; 28: 975-86.
20 .- Miyazaki S, Matsunaga T, Kawasaki K, Kobayashi I, Tada H, Yamaguchi K, et al. Separate isolation of Clostridium difficile spores and vegetative cells from the feces of newborn infants. Microbiol Immunol 1992; 36: 131-8.

21 .- Rolfe R D, Song W. Immunoglobulin and nonimmunoglobulin components of human milk inhibit Clostridium difficile toxin A-receptor binding. J Med Microbiol 1995; 42:10-9.

22 .- Tamma P D, Sandora T J. Clostridium difficile infection in children: current state and unanswered questions. J Pediatr Infect Dis Soc 2012; $1: 230-43$.

23 .- Morinville V, McDonald J. Clostridium difficileassociated diarrhea in 200 Canadian children. Can J Gastroenterol 2005; 19: 497-501.

24 .- Chopra T, Alangaden G J, Chandrasekar P. Clostridium difficile infection in cancer patients and hematopoietic stem cell transplant recipients. Expert Rev Anti Infect Ther 2010; 8: 1113-19.

25 .- Castañeda S, García A, Jaimes de la Hoz P, Jaramillo L, Perilla M A, Méndez M, et al. Enterocolitis asociada a enfermedad de Hirschsprung. Experiencia en un hospital universitario pediátrico. Cir Pediatr 2014; 27 : 78-83.

26.- Mezoff E, Mann E A, Hart K W, Lindsell C J, Cohen M B. Clostridium difficile infection and treatment in the pediatric inflammatory bowel disease population. J Pediatr Gastroenterol Nutr 2011; 52: 437-41.

27 .- Kelsen J R, Kim J, Latta D, Smathers S, McGowan K L, Zaoutis T, et al. Recurrence rate of Clostridium difficile infection in hospitalized pediatric patients with inflammatory bowel disease. Inflamm Bowel Dis 2011; 17: 50-5.

28.- Brown K A, Fisman D N, Moineddin R, Daneman R. The magnitude and duration of Clostridium difficile infection risk associated with antibiotic therapy: a hospital cohort Study. PLoS One 2014; 9: e105454.

29.- Surawicz C M, Brandt L J, Binion D G, Ananthakrishnan A N, Curry S R, Gilligan P H, et al. Guidelines for diagnosis, treatment, and prevention of Clostridium difficile infections. 
Am J Gastroenterol 2013; 108: 478-98.

30 .- Cohen S H, Gerding D N, Johnson S, Kelly C P, Loo V G, McDonald L C, et al. Clinical practice guidelines for Clostridium difficile infections in adults: 2010 update by the Society for Healthcare Epidemiology of America (SHEA) and the Infectious Diseases Society of America (IDSA). Infect Control Hosp Epidemiol 2010; 31: 431-55.

31 .- Suh KN, Gravel D, Mulvey MR. Clostridium difficile-associated infections in children admitted to acute care hospitals participating in the Canadian Nosocomial Infections Surveillance Program (CNISP), 2004-2005 [abstract 306]. In: Program of the 18th Annual Scientific Meeting of the Society for Healthcare Epidemiology of America (April 2008). The Society for Healthcare Epidemiology of America, Orlando, FL 2008.

32 .- Wolf L E, Gorbach S L, Granowitz E V. Extraintestinal Clostridium difficile: 10 years'experience at a tertiary-care hospital. Mayo Clin Proc 1998; 73: 943-7.

33 .- Huang S C, Yang Y L, Lee C T. Rectal prolapse in a child: an unusual presentation of Clostridium difficile associated pseudomembranous colitis. Pediatr Neonatol 2011; 52: 110-2.
34 .- Kusztrich A, Garten L, Hüseman D, Bührer C. Hemolytic uremic syndrome in a preterm infant. Pediatr Nephrol 2010; 25: 987-8.

35 .- Luna R A, Boyanton B L Jr, Mehta S, Courtney E M, Webb C R, Revell P, et al. Rapid stool-based diagnosis of Clostridium difficile infection by real-time PCR in a children's hospital. J Clin Microbiol 2011; 49: 851-7.

36.- Goldenberg J Z, Ma S S, Saxton J D, Martzen M R, Vandvik P O, Thorlund K, et al. Probiotics for prevention of Clostridium difficile-associated diarrea in adults and children. Cochrane Database Syst Rev 2013; 5: CD006095. 